\title{
An Unexpected Cause of Epigastric Pain
}

\author{
M Dykes, V Eze, A Taylor, S Shamshuddin
}

\section{CLINICAL PRESENTATION}

A 41 year-old female with previous history of bariatric surgery presented with a 2 day history of upper abdominal pain and vomiting. An abdominal radiograph (AXR) (Figure 1) and subsequent computer tomography (CT) were performed.

\section{IMAGES}
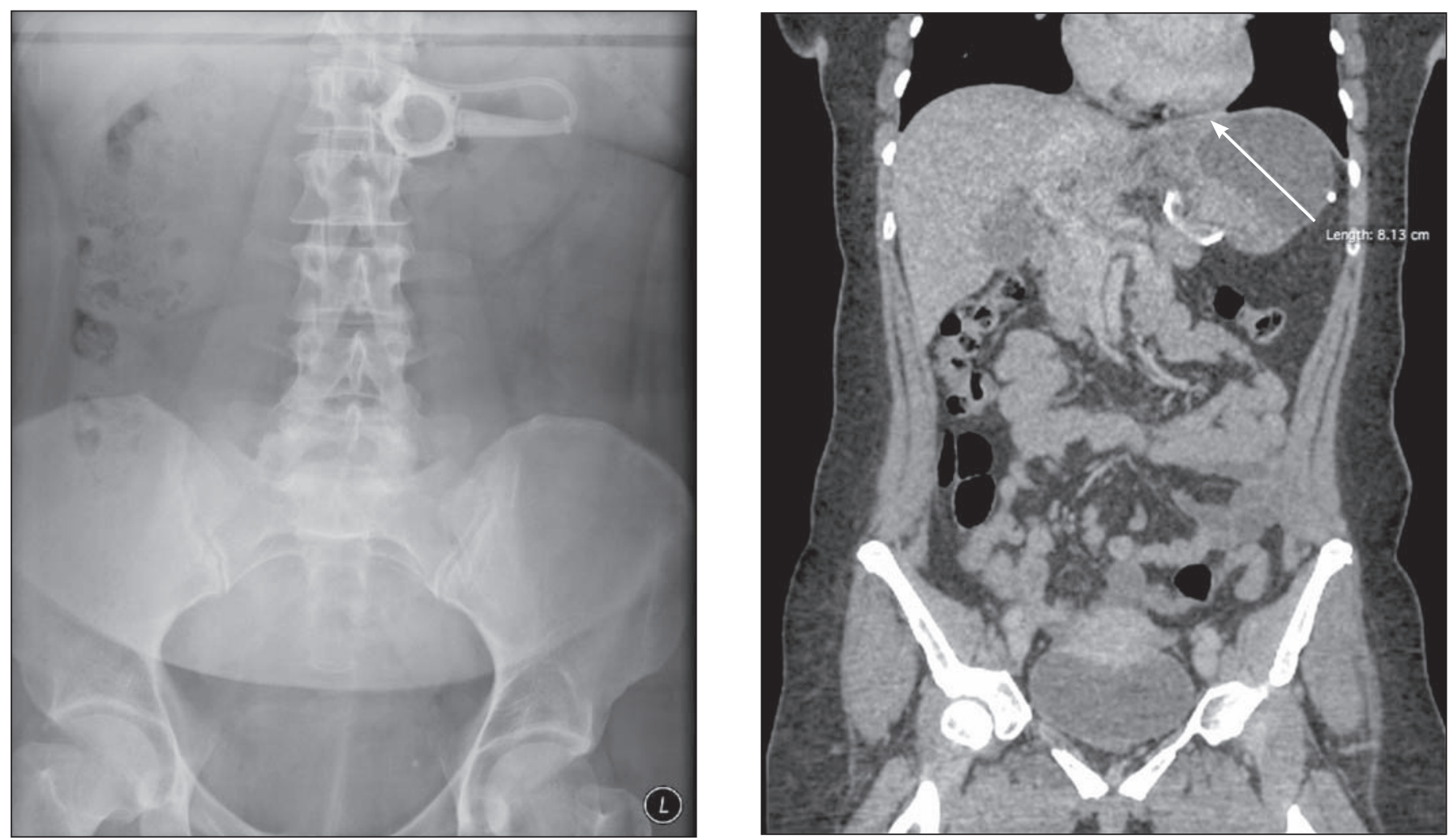

Figure 1: Initial AXR on admission demonstrating the subcutaneous port of the gastric band

\section{DIAGNOSIS}

Gastric band posterior slippage.

\section{TREATMENT}

In severe obstruction due to gastric band slippage, band decompression can alleviate gastric outlet symptoms. Ultimately correction of an uncomplicated slipped band to its original position laparoscopically is the best surgical intervention. ${ }^{1}$ Complicated slipped band scenarios can result in partial gastric resection due to acute gastric ischaemia as the vascular supply along the lesser curvature is stretched resulting in necrosis.

\section{RADIOGRAPHIC FEATURES}

Radiographic imaging including AXR, fluoroscopy and CT allows assessment of gastric band position and evaluation of complications.

Figure 2: Coronal CT image demonstrating an $8 \mathrm{~cm}$ proximal eccentric pouch dilatation

The normal band position on anteroposterior AXR is $5 \mathrm{~cm}$ below the left hemi-diaphragm. The band has a rectangular appearance and the $\phi$ (phi) angle, formed by the longitudinal axes of the spinal column and band, measures between 4 to 58 degrees. ${ }^{5}$ An obtuse $\phi$ (phi) angle greater than 58 degrees indicates an anterior slippage. An ovoid band appearance (the "O-sign") is characteristic of posterior band slippage. Water-soluble oral contrast upper gastrointestinal fluoroscopy can demonstrate the presence of an eccentric gastric pouch dilatation. A normal gastric pouch proximal to the band is less than $4 \mathrm{~cm}$ at its maximal diameter and empties within 20 minutes. CT imaging with oral contrast is used for evaluation of complications. Reformatted images allow review of the $\phi$ (phi) angle, proximal pouch calibre and components of the gastric band.

\section{GASTRIC BAND SLIPPAGE}

Laparoscopic gastric banding is a frequently used surgical treatment for morbid obesity. ${ }^{2}$ A small gastric pouch is 
created by positioning an adjustable silicone band $2 \mathrm{~cm}$ inferior to the gastroesophageal junction. A more distal position of the band increased risk to slippage. ${ }^{3}$ The band is adjusted by saline administration or withdrawal via a reservoir port connected to the band (Figure 3).

Patients can present with either acute symptoms of obstruction to chronic abdominal symptoms which include nocturnal vomiting, weight loss cessation or severe gastrooesophageal reflux.

Gastric band slippage arises when distal stomach distal to the band herniates proximally in either an anterior or posterior direction. This results in an abnormal band position and eccentric pouch dilatation. ${ }^{4}$ If unaddressed it can cause chronic stenosis.

\section{DIFFERENTIAL DIAGNOSES}

- Pouch dilatation. Symmetrical gastric pouch enlargement and delayed emptying maybe observed. Causes include stenosis, perigastric adhesions and nutritional overloading. ${ }^{3}$

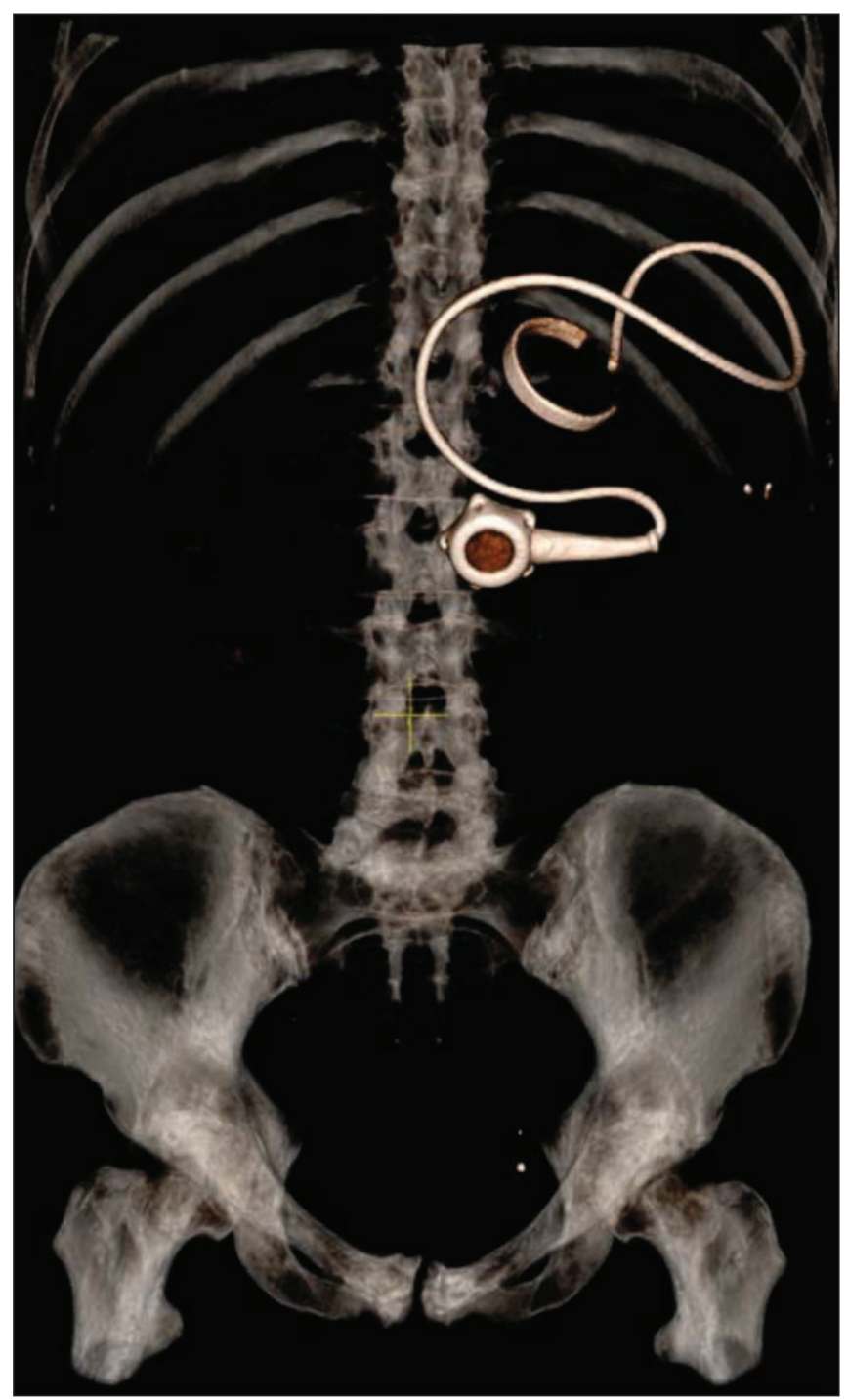

Figure 3: Adjustable gastric band components include a saline reservoir, connection tubing and circumferential band
- Intragastric erosion. Can be asymptomatic or present with vague epigastric pain, weight loss cessation or hematemesis. Causes include iatrogenic gastric wall injury, ischaemia secondary to band placement, inflammation or infection. ${ }^{6}$

- Oesophageal dysmotility. Patients can present with mild dysphagia, gastroesophageal reflux or aspiration. Cause can be due to insufficient dietary changes post procedure and longstanding gastroesophageal dysfunction.

- Inadequate band placement. A rare complication due to band placement at surgery within the perigastric fat or distal stomach. The latter results in gastric outlet obstruction.

\section{REFERENCES}

1. Wiesner W, Schöb O, Hauser RS, Hauser M. Adjustable laparoscopic gastric banding in patients with morbid obesity: radiographic management, results, and postoperative complications. Radiology 2000;216(2):389-394

2. Chandler RC, Srinivas G, Chintapalli KN, Schwesinger WH, Prasad SR. Imaging in bariatric surgery: a guide to postsurgical anatomy and common complications. AJR Am J Roentgenol 2008;190(1):122-135.

3. Weiner R, Bockhorn H, Rosenthal R, Wagner D. A prospective randomized trial of different laparoscopic gastric banding techniques for morbid obesity. Surg Endosc 2001;15(1):63-68.

4. Blachar A, Blank A, Gavert N, Metzer U, Fluser G, Abu-Abeid S. Laparoscopic adjustable gastric banding surgery for morbid obesity: imaging of normal anatomic features and postoperative gastrointestinal complications. AJR Am J Roentgenol 2007;188(2):472-479.

5. Mehanna MJ, Birjawi G, Moukaddam HA, Khoury G, Hussein M, Al-Kutoubi A. Complications of adjustable gastric banding, a radiological pictorial review. AJR Am J Roentgenol 2006;186(2):522-534.

6. Chisholm J, Kitan N, Toouli J, Kow L. Gastric band erosion in 63 cases: endoscopic removal and rebanding evaluated. Obes Surg 2011;21(11):1676-1681.

7. Wiesner W, Hauser M, Schöb O, Weber M, Hauser RS. Pseudo-achalasia following laparoscopically placed adjustable gastric banding. Obes Surg 2001; 11(4):513-518.

Images harvested from the RLI Museum of Cases, 2016. Department of Radiology, Royal Lancaster Infirmary.

Correspondence to: M Dykes

ST3 Radiology, RLI S. Shamshuddin Consultant Radiographer, RLI 